\title{
AKT3 and related molecules as potential biomarkers responsible for cryptorchidism and cryptorchidism-induced azoospermia
}

\author{
Hongshuai Jia ${ }^{1,2 \#}$, Tiantian $\mathrm{Ma}^{3 \#}$, Shujing Jia ${ }^{4}$, Yuru Ouyang ${ }^{1}$ \\ ${ }^{1}$ Department of Urology, Capital Institute of Pediatrics, Beijing, China; ${ }^{2}$ Department of Urology, Tianjin Children's Hospital, Tianjin, China; \\ ${ }^{3}$ Department of Nephrology, State Key Laboratory of Complex Severe and Rare Diseases, Peking Union Medical College Hospital, Chinese \\ Academy of Medical Science \& Peking Union Medical College, Beijing, China; ${ }^{4}$ Central Laboratory, the First Hospital of Handan, Handan, China \\ Contributions: (I) Conception and design: H Jia, T Ma; (II) Administrative support: H Jia; (III) Provision of study materials or patients: H Jia; (IV) \\ Collection and assembly of data: H Jia, T Ma; (V) Data analysis and interpretation: All authors; (VI) Manuscript writing: All authors; (VII) Final \\ approval of manuscript: All authors. \\ "These authors contributed equally to this work. \\ Correspondence to: Hongshuai Jia. Department of Urology, Capital Institute of Pediatrics, No. 2 Yabao Road, Chaoyang District, Beijing 100020, \\ China. Email: hongshuaijia@163.com.
}

Background: Cryptorchidism is a common congenital malformation strongly related to future oligospermia and male infertility. Normally functioning early-stage spermatogonia are vital to ensure fertility. The present study aimed to identify new differentially expressed genes (DEGs) associated with signaling pathways related to spermatogonial stem cell (SSC) maintenance during early spermatogenesis.

Methods: GEO2R was used to screen for genes differentially regulated in cryptorchidism using mRNA expression profiling data in the GEO database. DAVID was used to perform GO and KEGG enrichment analysis of DEGs to analyze their functions. A protein-protein interaction (PPI) network of DEGs was constructed using the STRING database. The hub genes in the PPI networks were identified using Maximal Clique Centrality (MCC) in Cytohubba, and the top 50 genes were displayed as hub genes using Cytoscape software. Then, the miRNAs targeting hub genes were predicted using miRWalk and an mRNA-miRNA interaction network was constructed using Cytoscape. We took the intersection of these target miRNAs and the differentially expressed miRNAs identified from a non-coding RNA sequencing dataset, GSE149084. Furthermore, the intersected miRNAs and their predicted target genes were validated in the testicular tissue of rats with cryptorchidism.

Results: A total of 474 DEGs were identified, most of which were annotated to the PI3K-AKT-mTOR signaling pathway. Hub genes related to the pathway were predicted to be targeted by 27 miRNAs. Further miRNA mining revealed that miRNA-7-5p and miRNA-519d-3p were both dysregulated in cryptorchidism patients. Further, we found that these two miRNAs were predicted with high confidence to share a common target gene, AKT3. In the testicular tissue of rats with cryptorchidism, miRNA-519d-3p was upregulated while miRNA-7-5p and AKT3 were downregulated. We also found that AKT3 plays an essential role in regulating SSC state through the PI3K-AKT-mTOR signaling pathway and that AKT3 is one of the key genes related to SSC self-renewal, proliferation, and differentiation.

Conclusions: The PI3K-AKT-mTOR signaling pathway functions in SSC maintenance, and alterations in this pathway may explain defects in spermatogenesis. AKT3-related miRNAs, including hsa-miR-7-5p and hsa-miR-519d-3p, might be responsible for cryptorchidism and cryptorchidism-induced azoospermia and serve as potential biomarkers.

Keywords: Cryptorchidism; azoospermia; bioinformatic analysis; biomarkers; miRNA

Submitted Jan 24, 2021. Accepted for publication Jun 01, 2021.

doi: $10.21037 / \mathrm{tp}-21-31$

View this article at: https://dx.doi.org/10.21037/tp-21-31 


\section{Introduction}

Cryptorchidism or undescended testis (UDT) is the most common congenital malformation in male infants, affecting $1.0-4.6 \%$ of full-term and $1.1-45.0 \%$ of premature newborns (1). The occurrence of cryptorchidism is closely associated with many factors, including genetic, environmental, mechanical, anatomical, and numerous endocrine factors. Cryptorchidism can be treated by early surgery to improve the appearance. Cryptorchidism is the key accepted risk factor for malignancy of the testis, carrying a relative risk between 2.2 and 3.8 times higher than the background population according to a recent metaanalysis (2). Early Orchidopexy may even lower the risk of developing testicular cancer (3). However, surgery does not appear to fix pre-existing pathological lesions, and some patients still exhibit lower fertility as adults $(4,5)$. Previous studies have reported that $13 \%$ of children with unilateral cryptorchidism and $89 \%$ of children with untreated bilateral cryptorchidism have azoospermia. Regrettably, $10 \%$ of patients with unilateral cryptorchidism and $32 \%$ of patients with bilateral cryptorchidism still exhibit a high incidence of infertility after treatment (6).

Low levels of adult dark spermatogonia (Ad spermatogonia) or spermatogonial stem cells (SSCs) were found in testicular biopsies of UDTs in boys younger than two years old. Low numbers of SSCs are strongly associated with future oligospermia, and insufficient numbers of $\mathrm{Ad}$ spermatogonia are considered a putative cause of infertility (7-9). Sperm production depends on the SSC pool, which is formed through the differentiation of precursor cells (gonocytes) in infancy. Throughout adulthood, SSCs renew or differentiate to maintain stem cell reserves and provide cells for the spermatogenic cycle (10). For a long time, scientists have mostly focused on spermatogenesis after puberty in children. The early stages of germ cell development have only gradually received attention in recent years, with studies revealing genes and processes that regulate SSC self-renewal, proliferation, and differentiation (11). Currently, little is known about the mechanisms regulating the proliferation and differentiation of SSCs. Because abnormal proliferation and differentiation of SSCs may lead to infertility in adults, it is essential to further explore the molecular mechanisms underlying the regulation of these processes.

miRNAs, a class of non-coding RNA molecules that are approximately 22 nucleotides in length and do not code for proteins, are closely associated with the development and prognosis of cryptorchidism $(12,13)$. More than $50 \%$ of human genes are regulated by miRNAs, which bind to the 3'-untranslated regions (3'-UTRs) of mRNAs and thereby downregulate gene expression. Recent studies have demonstrated the importance of miRNAs to SSC and spermatid differentiation $(14,15)$. Total loss of miRNA function represses spermatogenesis. Emerging evidence shows that miRNAs are involved in germ cell development and that they are expressed at higher or lower levels during the early stages of spermatogenesis compared with later stages $(16,17)$.

Because miRNAs play important roles in spermatogenesis, we also identified miRNAs potentially targeting the differentially expressed genes (DEGs). The present study aimed to identify new DEGs associated with cryptorchidism and to find susceptibility genes related to SSC maintenance during early spermatogenesis. These findings may provide insight into new directions for studying the pathogenesis of cryptorchidism and cryptorchidism-induced azoospermia.

We present the following article in accordance with the MDAR reporting checklist (available at https://dx.doi. org/10.21037/tp-21-31).

\section{Methods}

\section{Microarray analysis of cryptorchidism patients}

The study was conducted in accordance with the Declaration of Helsinki (as revised in 2013). Two gene expression datasets generated from clinical samples of cryptorchidism patients were retrieved from the GEO database [GSE16191 (18) and GSE25518 (6); Affymetrix GPL570 platform, Affymetrix Human Genome U133 Plus 2.0 Array]. The GSE16191 dataset comprises gene expression data for 14 UDT and 4 contralateral descended testis (DT) tissue samples from testicular agenesis patients, while the GSE25518 is for 19 UDT and 4 contralateral DT tissue samples from testicular agenesis patients. All of the human data evaluated in the present study were downloaded from a public database. Note that the probes of the Affymetrix GPL570 chip were automatically converted into gene symbols according to the current annotations.

\section{Identification of DEGs}

The DEGs between the UDT and DT tissue samples were identified using GEO2R, a tool that allows users to compare two or more groups of GEO samples (19). Probe 
sets without corresponding gene symbols were removed from analysis. When a single gene matched multiple probe sets, the values from the probe sets were averaged.

\section{Functional enrichment analysis of DEGs}

The Database for Annotation, Visualization and Integrated Discovery (DAVID, version 6.8), which allows integration of functional annotation information with analysis tools (20), was used to perform Encyclopedia of Genes and Genomes (KEGG) and Gene Ontology (GO) enrichment analysis of the DEGs. The Cytoscape (version 3.7.2) plug-in ClueGO (v. 2.5.7) (21) was used to decipher the pathway networks and determine the biological functions of DEGs. Each gene set was annotated using KEGG pathway profiles.

\section{Protein-protein interaction (PPI) network construction}

The PPI networks of DEGs were predicted using the Search Tool for the Retrieval of Interacting Genes (STRING, version 11.0) (22) to provide insights into the mechanisms of cryptorchidism development. A combined score of $>0.4$ was considered statistically significant. The results of STRING analysis were imported into Cytoscape to visualize interaction networks.

\section{Identification of hub genes}

The Cytoscape plug-in Cytohubba (23) was used to identify hub genes and sub-networks in the DEG PPI networks. The hub genes in the PPI networks were identified using Maximal Clique Centrality (MCC), which performs better on PPI networks than the other 11 available methods in Cytohubba (24), and the top 50 genes were displayed as hub genes using Cytoscape software. STRING was used to draw the hub genes in the PPI network, and the biological processes and pathways were further analyzed by DAVID.

\section{Further miRNA mining}

The miRNAs targeting hub genes were predicted using miRWalk 2.0 (25). To verify the accuracy of the results, the overlap of the miRWalk 2.0 predictions with those of the TargetScan, miRDB, miRTarBase, and miRWalk databases was determined. The criteria for selecting these miRNAs were as follows: $\mathrm{P}<0.05$, the target binding site is in the 3'-UTR, and the minimum seed sequence length is 7 . We took the intersection of these target miRNAs and the differentially expressed miRNAs (DE-miRNAs) identified from the GSE149084 RNA sequencing (RNA-seq) dataset. The overlapping targets were further processed to construct an mRNA-miRNA interaction network using Cytoscape.

\section{Validation of the predicted miRNAs and construction of an mRNA-miRNA interaction network}

To verify the mRNA-miRNA interaction network, we used the genome-wide transcriptional profiles of UDT and DT tissue samples (GSE149084). In order to check the difference between the genome-wide transcriptional profile of children with cryptorchidism and in normal control testis, GSE149084 dataset examined testicular tissue including the samples of 6 cases of children with cryptorchidism and 2 cases of testicular trauma children and 1 case of incarcerated inguinal hernias. The transcriptome sequencing of the 9 testicular tissues was performed by RNA-seq technique. Testicular tissue samples from six children with UDTs and three with DTs were separately analyzed. DE-miRNAs were identified and then compared with the miRNAs targeting hub genes.

\section{Animals}

A total of 20 male SD rats, aged 3-4 weeks, were obtained from Beijing Vital River Laboratory Animal Technology Co., Ltd. (Beijing, China). All rats were maintained in a controlled environment set at $25 \pm 5{ }^{\circ} \mathrm{C}$ and $30-70 \%$ humidity with a $12 \mathrm{~h} / 12 \mathrm{~h}$ light/dark cycle. Food and water were provided ad libitum. Experiments were performed under a project license (No.: SHERLL2019025) granted by the Ethics Committee of the Children's Hospital of Capital Institute of Pediatrics, in compliance with the guidelines for the care and use of animals.

\section{The rat model of cryptorchidism}

A total of 20 male SD rats were randomly divided into a cryptorchidism group $(\mathrm{n}=10)$ and a control group $(\mathrm{n}=10)$. The 3-week-old rats we purchased were subjected to adaptive observation for one week, subjected to the modelinduction surgery at the age of 4 weeks, and were then sacrificed for removal of testicular tissue after another 3 weeks. Surgery was performed under 3\% pentobarbital $(0.15 \mathrm{~mL} / 100 \mathrm{~g})$ anesthesia. The skin of the middle-lower abdomen was disinfected and a midline abdominal incision was made. The right testis was pulled into the abdominal 
cavity and sutured to the abdominal wall, while the left testis was maintained in the scrotum. The right testis of the control group rats was subjected to sham surgery.

\section{RNA extraction}

Cryptorchidism tissue was selected randomly from the 20 rats in the 2 groups and frozen in liquid nitrogen. Total RNA was extracted using the mirVana ${ }^{\mathrm{TM}}$ RNA Isolation Kit (AM1561) according to the manufacturer's instructions. RNA yield was determined using a NanoDrop 2000 spectrophotometer (Thermo Scientific, USA), and integrity by agarose gel electrophoresis followed by ethidium bromide staining.

\section{Quantitative reverse transcription polymerase chain reaction ( $q R T-P C R)$}

The primers were designed based on mRNA sequences obtained from the NCBI database or microRNA sequences obtained from the miRBase database (Release 20.0). Primers were synthesized by TsingKe Biotech. Reverse transcription was performed in a GeneAmp ${ }^{\circledR}$ PCR System 9700 (Applied Biosystems), and real-time PCR was performed using a LightCycler $^{\circledR} 480$ II Real-time PCR Instrument (Roche). The expression levels of mRNAs and miRNAs were normalized to $\beta$-actin and U6, respectively, and differences in relative expression were calculated using the $2^{-\Delta \Delta \mathrm{Ct}}$ method.

\section{Statistical analysis}

$\mathrm{P}$ values were adjusted using the Benjamini-Hochberg procedure to decrease the false discovery rate. Probe sets without corresponding gene symbols were removed from analysis. The following selection criteria were used to define DEGs: at least a 2.0-fold change between UDT and normal DT tissue samples and a $\mathrm{P}$ value $<0.01$. To analyze the function of DEGs, biological analyses were performed using DAVID online database. $\mathrm{P}<0.05$ was considered statistically significant. The following criteria were used to screen for DE-miRNAs in selected GEO datasets: $\log _{2} \mathrm{FC}>1$ or $<-1$ and $\mathrm{P}<0.01$.

\section{Results}

\section{Identification of DEGs associated with cryptorchidism}

Genes differentially expressed in cryptorchidism were identified by comparing gene expression in UDT and DT tissues. A total of 682 DEGs in GSE16191 and 575 DEGs in GSE25518 were identified after the standardization of the microarray results; 474 DEGs were identified in both datasets (Figure 1A), 343 of which were upregulated in UDT tissues compared with DT tissues and 131 of which were downregulated.

\section{PPI network construction}

A PPI network of DEGs was constructed (Figure 1B), and the top 50 nodes according to MCC calculations of connectivity were designated as hub genes (Figure 1C). The STRING online platform was utilized to analyze the network of the hub genes and their co-expressed genes.

\section{Functional enrichment analysis of DEGs}

DAVID was used for functional and pathway enrichment analysis of the DEGs to determine their biological functions. GO analysis showed that DEGs were significantly enriched in the biological process categories peptidyl-tyrosine dephosphorylation, negative regulation of cell proliferation, and intracellular signal transduction (Figure 2A). DEGs were mainly enriched in the cell component categories nucleoplasm, cytosol, and membrane (Figure 2B). And DEGs were primarily enriched in the molecular function categories protein binding and protein tyrosine phosphatase activity (Figure 2C). KEGG pathway analysis revealed that the DEGs were mainly enriched in the spliceosome, PI3KAKT, and mTOR signaling pathways (Figure 2D). Cytoscape ClueGo enrichment tests indicated that the DEGs were mainly enriched in negative regulation of cell adhesion and proliferation, negative regulation of developmental process, cell junction assembly, ameboidal-type cell migration, and regulation of cell cycle process, as shown in Figure 3. The results of functional enrichment analysis of the hub genes are shown in Figure 4. The enriched GO categories and KEGG pathways for hub genes were basically the same as those for all DEGs. The DEGs in the UDT samples were mainly enriched in cellular processing and the PI3K-AKT-mTOR signaling pathway.

\section{Further miRNA mining}

Hub genes related to the PI3K-AKT-mTOR pathway, which participates in SSC self-renewal and differentiation, were identified, and miRWalk 2.0 software was used to investigate mRNA-miRNA interactions. The miRNAs 
A

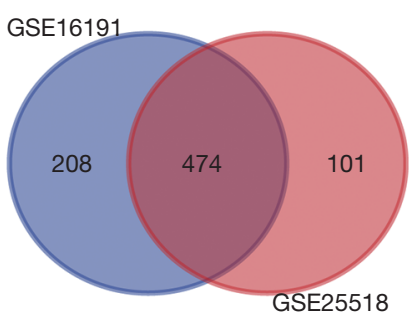

C

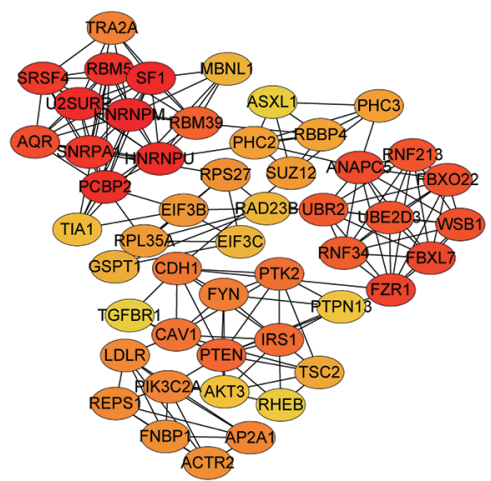

B

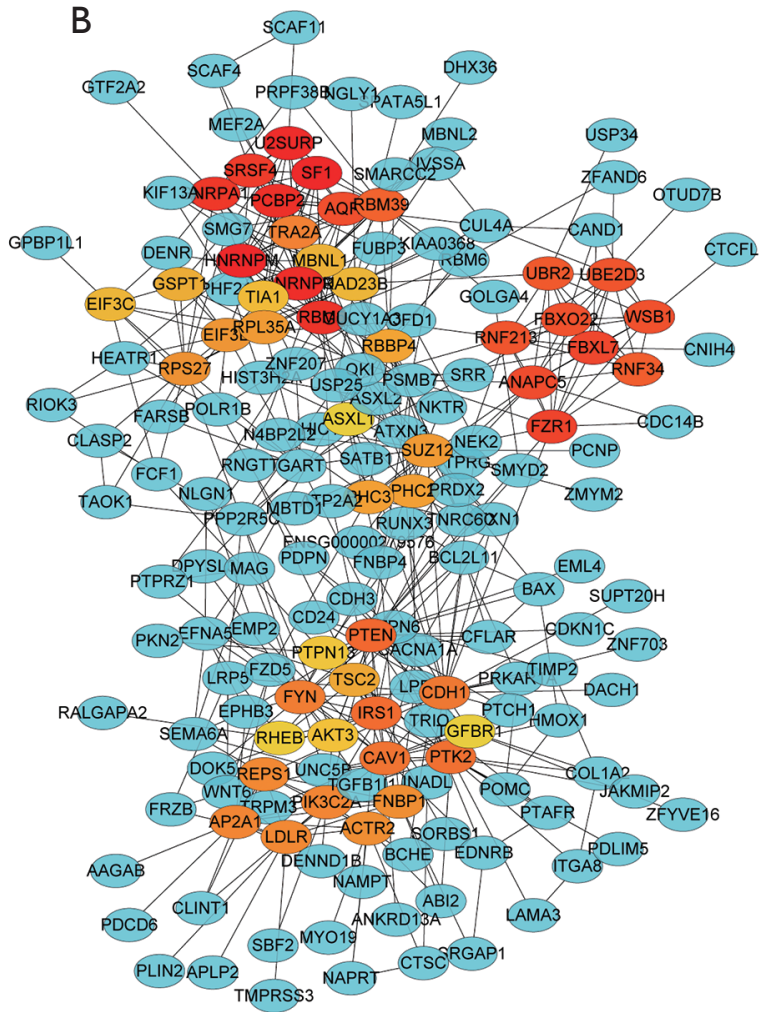

Figure 1 Venn diagram, PPI network and the hub genes of DEGs. (A) DEGs were selected with a fold change $>2$ and $\mathrm{P}$ value $<0.01$ among the mRNA expression profiling sets GSE16191 and GSE25518. The 2 datasets showed an overlap of 474 genes. (B) The part of PPI network of DEGs was constructed using Cytoscape. (C) The hub genes in the PPI networks were identified according to calculating the nodes MCC of connectivity, and the top 50 hub genes were displayed in the Cytoscape software. PPI, protein-protein interaction; DEG, differentially expressed gene; MCC, Maximal Clique Centrality.

predicted by the miRWalk, TargetScan, miRDB, and miRTarBase databases were selected as miRNAs that potentially target hub genes. Further miRNA mining results showed that miRNA-7-5p and miRNA-519d-3p were both dysregulated in cryptorchidism patients.

\section{Validation of the predicted miRNAs and mRNA-miRNA interaction network analysis}

Forty-nine DE-miRNAs, including 24 upregulated (Table 1) and 25 downregulated genes (Table 2), were found in analysis of the GSE149084 profile (Figure 5A). DE-miRNAs that overlapped with our predicted pivotal miRNAs targeting hub genes were selected as candidate DE-miRNAs (Figure 5B). The overlapping DE-miRNAs were hsa-miR-7-5p and hsa-miR-519d-3p. By drawing mRNA-miRNA interaction networks, we found that the DE-miRNAs shared a common target gene, AKT3 (Figure 6).

\section{Verification of potential biomarker expression by qRT-PCR}

The differential expression of the biomarkers miRNA$7-5 p$ and miRNA-519d-3p was validated in the testicular tissue of cryptorchidism rats using qRT-PCR analysis. The results showed that the expression levels of miRNA-7-5p $(\mathrm{P}=0.022)$ and AKT3 $(\mathrm{P}<0.001)$ in the testicular tissue of cryptorchidism rats were obviously lower than those in the sham surgery controls. However, miRNA-519d-3p was upregulated $(\mathrm{P}$ value $=0.002)$, contrary to the sequencing results (Figure 7).

\section{Discussion}

Cryptorchidism is a common congenital malformation strongly associated with future oligospermia and male infertility (26). Early-stage spermatogonia ensure a constant supply of spermatocytes, which is essential for 
A

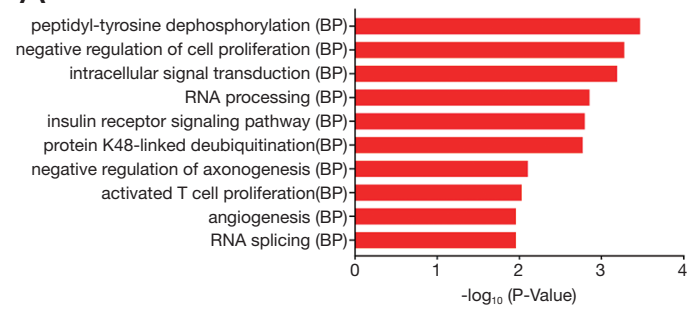

C

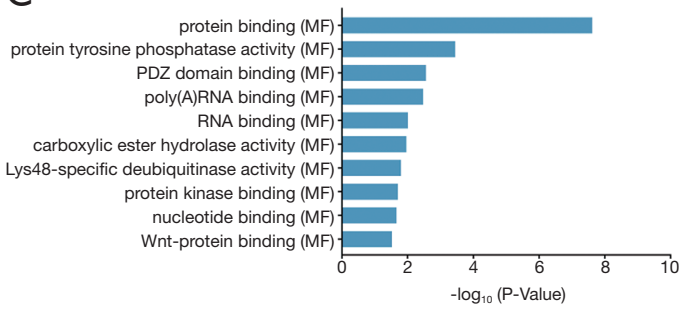

B
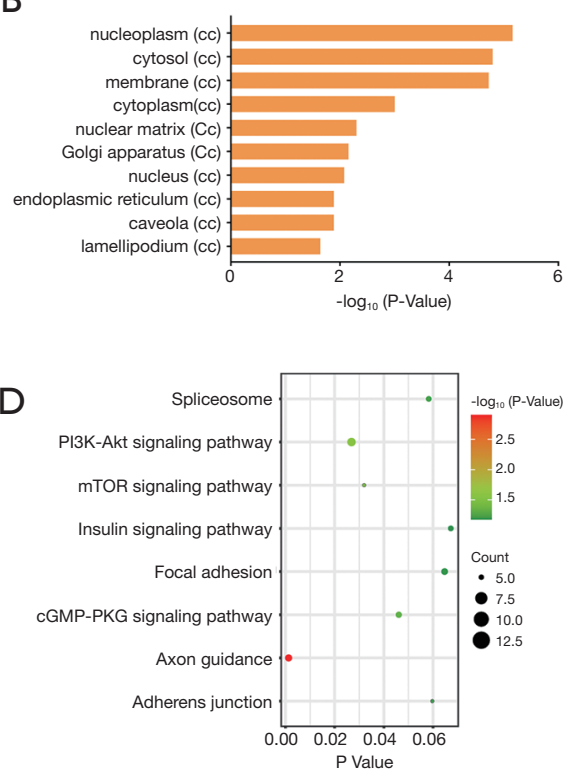

Figure 2 Top 10 significant enrichment GO and KEGG terms of DEGs. (A) BP: biological process; (B) CC: cellular component; (C) MF: molecular function; (D) KEGG: signaling pathway. GO, Gene Ontology; DEG, differentially expressed gene.

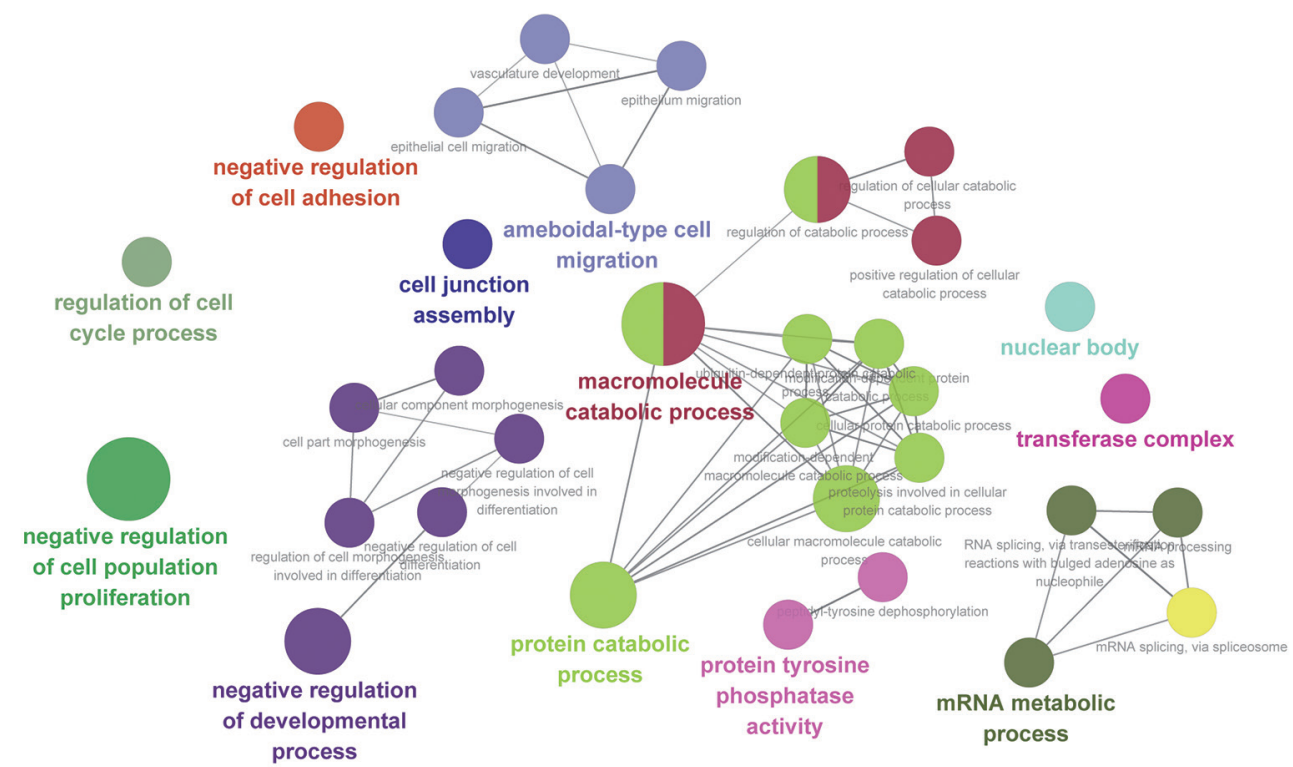

Figure 3 ClueGO was utilized to decipher the pathways network and determine their biological functions for the DEGs. DEG, differentially expressed gene.

fertility. Gene expression must be precisely controlled transcriptionally or posttranscriptionally to maintain the function of spermatocytes (27). In the present study, two mRNA microarray datasets were analyzed to identify DEGs between UDT and DT tissue. DAVID and ClueGo enrichment analysis of the 474 identified DEGs showed that these genes were mainly annotated to the PI3K-AKTmTOR signaling pathway, which is involved in cellular processing. This suggests that the PI3K-AKT-mTOR signaling pathway, which regulates the maintenance 


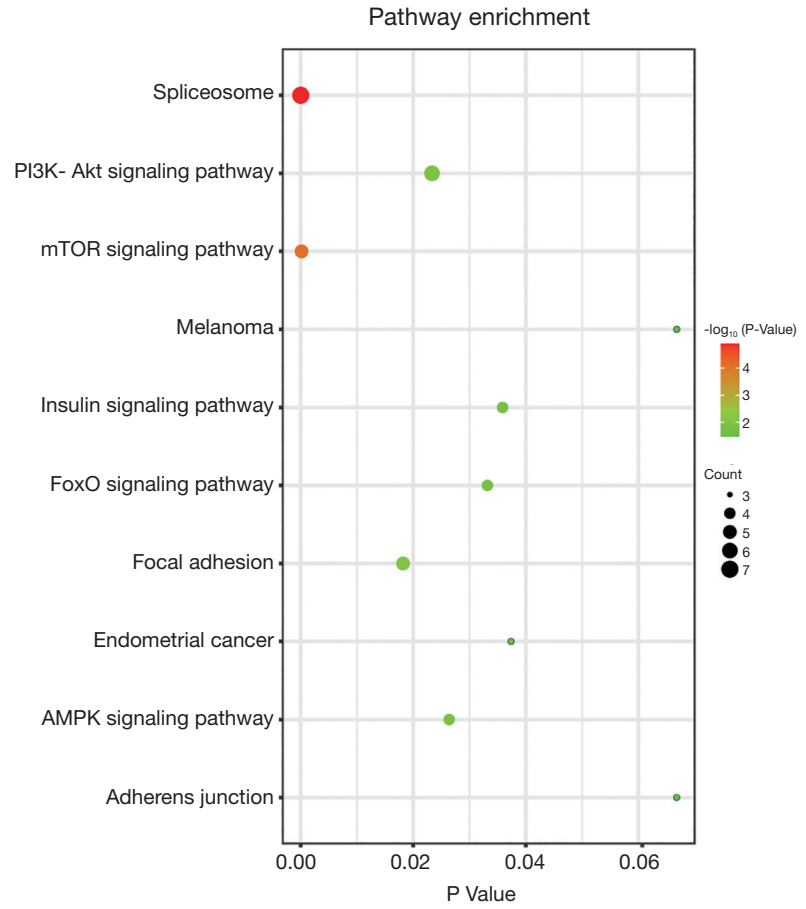

Figure 4 Biological analysis of hub genes showed the top 10 KEGG enrichment pathways.

of SSCs, is critical for cryptorchidism. Therefore, we identified molecules with significant relevance to AKT3, including miR-7-5p and miR-519d-3p, to investigate potential mechanisms underlying the relationship between cryptorchidism and cryptorchidism-induced azoospermia. We believe that based on the regulatory networks constructed for these molecules, the PI3K-AKT-mTOR signaling pathway is affected in cryptorchidism.

Before focusing on the molecular genetics data of our study, it should be noted that the etiopathology of cryptorchidism is complex, with a wealth of data from many studies having implicated a variety of genetic, maternal, and environmental risk factors (28). Physiologically, it is known that correct testicular descent depends on the hypothalamic-pituitary-gonadal axis. Epidemiologically, the increased risk of cryptorchidism in first-degree relatives suggests a significant genetic contribution to susceptibility. Although there are reported mutations in insulin-like 3 and the relaxin/insulin-like family peptide receptor 2 linked to nonsyndromic cryptorchidism, no pathogenic impacts of these genetic variants has been established. Regarding material influences, and among multiple other reported factors, low birth weight and retardation of intrauterine
Table 1 List of 24 upregulated miRNAs of testes tissue in cryptorchidism patients from database GSE149084

\begin{tabular}{|c|c|c|}
\hline miRNA & $P$ value & $\mathrm{FC}$ \\
\hline hsa-miR-8085 & $1.71 \times 10^{-04}$ & 20.36 \\
\hline hsa-miR-3621 & $4.20 \times 10^{-04}$ & 18.06 \\
\hline hsa-miR-10524-5p & $5.06 \times 10^{-03}$ & 17.03 \\
\hline hsa-miR-6789-3p & $4.66 \times 10^{-03}$ & 10.05 \\
\hline hsa-miR-4712-3p & $5.43 \times 10^{-03}$ & 6.12 \\
\hline hsa-miR-4680-5p & $7.11 \times 10^{-03}$ & 5.42 \\
\hline hsa-miR-3976 & $7.94 \times 10^{-03}$ & 4.31 \\
\hline hsa-miR-4287 & $4.29 \times 10^{-04}$ & 3.90 \\
\hline hsa-miR-3141 & $1.48 \times 10^{-03}$ & 3.62 \\
\hline hsa-miR-4433a-3p & $3.95 \times 10^{-03}$ & 3.54 \\
\hline hsa-miR-1199-5p & $2.97 \times 10^{-03}$ & 3.26 \\
\hline hsa-miR-5588-5p & $3.47 \times 10^{-04}$ & 3.18 \\
\hline hsa-miR-4700-5p & $5.61 \times 10^{-04}$ & 2.88 \\
\hline hsa-miR-7108-3p & $8.04 \times 10^{-04}$ & 2.79 \\
\hline hsa-miR-6872-3p & $2.65 \times 10^{-04}$ & 2.76 \\
\hline hsa-miR-3611 & $1.74 \times 10^{-05}$ & 2.70 \\
\hline hsa-miR-3160-5p & $5.27 \times 10^{-03}$ & 2.62 \\
\hline hsa-miR-3195 & $2.03 \times 10^{-03}$ & 2.46 \\
\hline hsa-miR-5088-5p & $2.42 \times 10^{-04}$ & 2.39 \\
\hline hsa-miR-6748-5p & $9.24 \times 10^{-03}$ & 2.38 \\
\hline hsa-miR-219a-1-3p & $6.14 \times 10^{-03}$ & 2.37 \\
\hline hsa-miR-11401 & $3.23 \times 10^{-03}$ & 2.34 \\
\hline hsa-miR-615-3p & $3.43 \times 10^{-03}$ & 2.23 \\
\hline hsa-miR-3150b-5p & $1.99 \times 10^{-03}$ & 2.00 \\
\hline
\end{tabular}

FC, fold change.

growth have been strongly associated with cryptorchidism, and there is additional evidence suggesting that maternal smoking, alcohol use, and gestational diabetes increase this risk (29). Reports of associations with both sex hormone imbalance and exposure to endocrine disrupting chemicals merit further investigations, which should be facilitated by the gene network that we generated in the present study. Finally, there are also some fascinating trends in the clinical data suggesting that in vitro fertilization and even twinning are associated with cryptorchidism. Our study's implication of the PI3K-AKT-mTOR signaling in 
Table 2 List of 25 downregulated miRNAs of testes tissue in cryptorchidism patients from database GSE149084

\begin{tabular}{|c|c|c|}
\hline miRNA & $P$ value & $\mathrm{FC}$ \\
\hline hsa-miR-519b-5p & $1.82 \times 10^{-03}$ & 0.14 \\
\hline hsa-miR-520f-3p & $8.10 \times 10^{-04}$ & 0.18 \\
\hline hsa-miR-518e-3p & $3.55 \times 10^{-05}$ & 0.20 \\
\hline hsa-miR-519a-3p & $7.26 \times 10^{-03}$ & 0.21 \\
\hline hsa-miR-517c-3p & $5.98 \times 10^{-04}$ & 0.22 \\
\hline hsa-miR-519d-3p & $6.17 \times 10^{-05}$ & 0.23 \\
\hline hsa-miR-518a-3p & $7.93 \times 10^{-03}$ & 0.24 \\
\hline hsa-miR-518c-3p & $5.28 \times 10^{-03}$ & 0.26 \\
\hline hsa-miR-1283 & $8.81 \times 10^{-03}$ & 0.26 \\
\hline hsa-miR-515-3p & $6.75 \times 10^{-03}$ & 0.27 \\
\hline hsa-miR-526b-5p & $5.39 \times 10^{-03}$ & 0.27 \\
\hline hsa-miR-539-5p & $3.31 \times 10^{-03}$ & 0.27 \\
\hline hsa-miR-517b-3p & $4.23 \times 10^{-03}$ & 0.27 \\
\hline hsa-miR-1299 & $2.33 \times 10^{-03}$ & 0.28 \\
\hline hsa-miR-518b & $1.96 \times 10^{-03}$ & 0.28 \\
\hline hsa-miR-516b-5p & $4.28 \times 10^{-03}$ & 0.28 \\
\hline hsa-miR-515-5p & $3.23 \times 10^{-03}$ & 0.29 \\
\hline hsa-miR-517a-3p & $1.63 \times 10^{-03}$ & 0.29 \\
\hline hsa-miR-520c-3p & $2.85 \times 10^{-03}$ & 0.30 \\
\hline hsa-miR-3059-5p & $1.60 \times 10^{-03}$ & 0.32 \\
\hline hsa-miR-520a-3p & $5.16 \times 10^{-03}$ & 0.36 \\
\hline hsa-miR-7-5p & $1.42 \times 10^{-03}$ & 0.40 \\
\hline hsa-miR-155-5p & $1.64 \times 10^{-03}$ & 0.40 \\
\hline hsa-miR-3529-3p & $2.41 \times 10^{-03}$ & 0.40 \\
\hline hsa-miR-224-5p & $2.25 \times 10^{-03}$ & 0.48 \\
\hline
\end{tabular}

FC, fold change.

dysregulated testicular descent could be further explored using for example with IVF experiments using knockout (or conditional knockout) animals.

The increased temperature in UDT might also change the signals activating the PI3K-AKT-mTOR signaling pathway, and thus inhibit SSC self-renewal. Several studies have reported that dysregulation of the cycle of SSC proliferation and differentiation plays an essential role in the azoospermia (11). Interestingly, scholars found in SSC transplantation experiments that activation of H-RAS was sufficient to induce SSC self-renewal, and that PI3K/AKT was a RAS effector. Activation of the PI3K-AKT pathway was found to induce the logarithmic proliferation of GS cells for at least 5 months (30). Similarly, other studies showed that PI3K/AKT regulates SSC self-renewal by upregulating the ETV5, Bcl6b, and LHX1 genes $(31,32)$. In addition, the PI3K-AKT-mTOR signaling pathway was found to be induced by exogenous stimuli (33), suggesting that the increased temperature in UDT in vivo might affect SSC function. These results indicate that PI3K-AKT activation plays a vital role in the self-renewal of SSCs and that the downregulation of AKT3 in the testicular tissue of cryptorchidism rats might indicate defects in SSC selfrenewal.

Our gene enrichment analysis showed that the PI3KAKT, mTOR, FOXO, AMPK, and insulin signaling pathways might have close interactions with cryptorchidism. Insulin-like growth factor 1 activates its receptors as well as the PI3K-AKT-mTOR signaling pathway, which causes FoxO1 to leave the nucleus and enter the cytoplasm (34). The departure of FoxO1 activates the androgen receptor, leading to the initiation of cell proliferation, lipid synthesis, and inflammation (35). It is already known that gonocyte transformation into spermatogonia via proliferation and migration to the basement membrane is delayed in UDTs. Also, spermatogonia always remain on the basement membrane during the continuous proliferation and differentiation of the vas deferens (36). Therefore, the balance between AKT3 and PI3K-AKT-mTOR is essential for maintaining SSC function.

We analyzed genes related to the PI3K-AKT-mTOR pathway to identify their importance in maintaining SSC function and their relationships with key genes captured in the mRNA-miRNA network. Further, mRNA-miRNA interaction networks showed that miRNA-7-5p and miRNA519d-3p share a common target gene, AKT3. Meanwhile, PPI network analysis showed that of the core upstream regulators, AKT3 is highly upregulated in UDT tissue and likely plays an important role in SSC maintenance. Previous studies indicated that AKT3 could regulate cell proliferation, survival, and migration signal transduction, and that it is essential for the multipotency of stem cells, including human SSCs (37). Consistent with this, AKT3 knockdown was found to result in a significantly lower cell survival rate, an increased number of G1 phase cells, and a decreased number of $S$ and G2/M phase cells (38).

Increased levels of intracellular non-phosphorylated AKT3 might accelerate the tissue damage caused by 
A

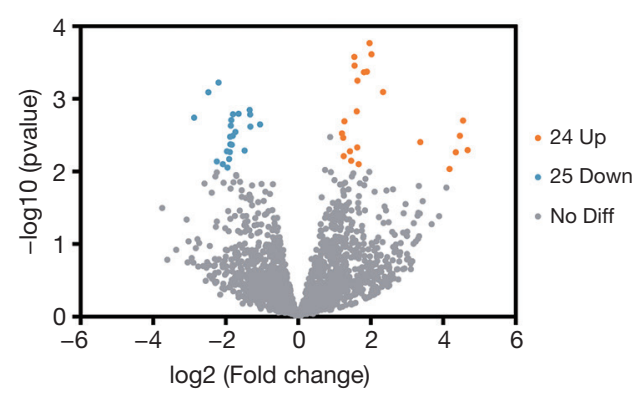

B

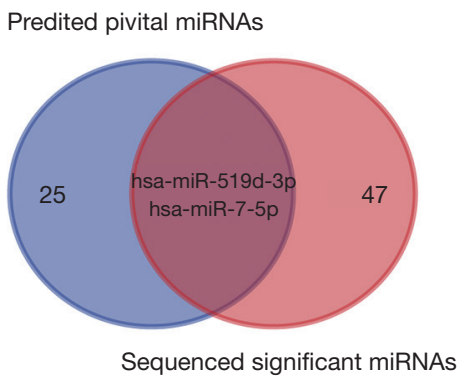

Figure 5 Validation of the predicted pivotal miRNAs by RNA-seq from the GEO database. (A) Differentially expressed genes in UDTs and DTs samples from GSE149084 of the GEO database were shown in the volcano plot, with blue dots representing significantly downregulated genes in UDTs samples and orange dots representing significantly upregulated genes. (B) Differential expression miRNAs from GSE149084 were intersected with our prediction miRNAs of hub genes. UDTs, undescended testis; DTs, descended testis.

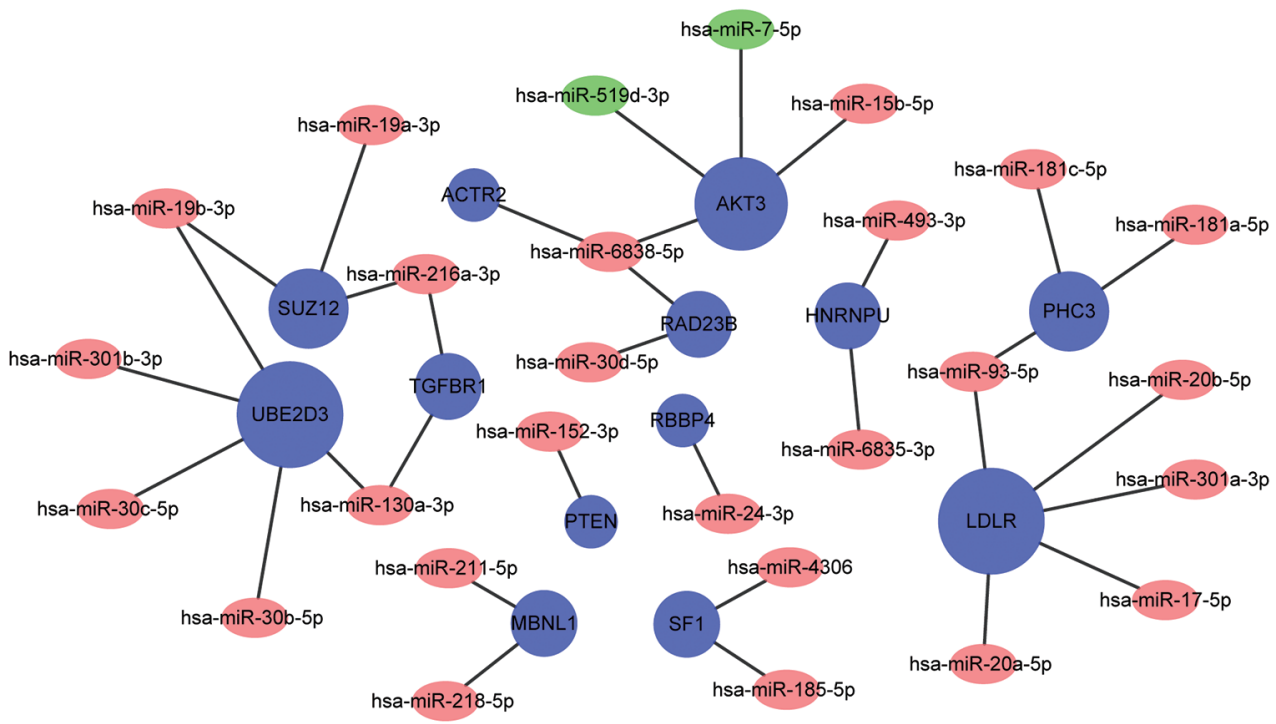

Figure 6 Interaction network between hub genes and its targeted miRNAs. Genes were colored in blue, and node size was adjusted according to the number of targeted miRNAs; miRNAs were colored in red. The two intersected miRNAs between differentially expressed miRNAs from GSE149084 and predicted miRNAs of hub genes were colored in green.

cryptorchidism. In fact, in the early stages of UDT, AKT3 was found to activate the transcription of downstream anti-apoptotic factors through PI3K-AKT-mTOR phosphorylation (39). PI3K is a dimer composed of regulatory subunit $\mathrm{p} 85$ and catalytic subunit $\mathrm{p} 110$. When PI3K binds to growth factor receptors, it changes the protein structure of AKT and activates it. Then a series of downstream substrates such as apoptosis-related proteins Bad and Caspase9 are activated or inhibited by phosphorylation, leading to the regulation of cell proliferation, differentiation, apoptosis, and migration (39). However, with an extended duration of UDT, the levels of intracellular non-phosphorylated AKT3 protein increase. These non-phosphorylated AKT3 proteins could inhibit the phosphorylated kinase PI3K signal transduction pathway and enhance the expression of the apoptotic proteins Bad and Caspase9, disrupting the stability of the SSCs, spermatogonia self-renewal, and spermatogenesis (40). Therefore, we hypothesize that AKT3 might be crucial for SSC self-renewal and differentiation. 

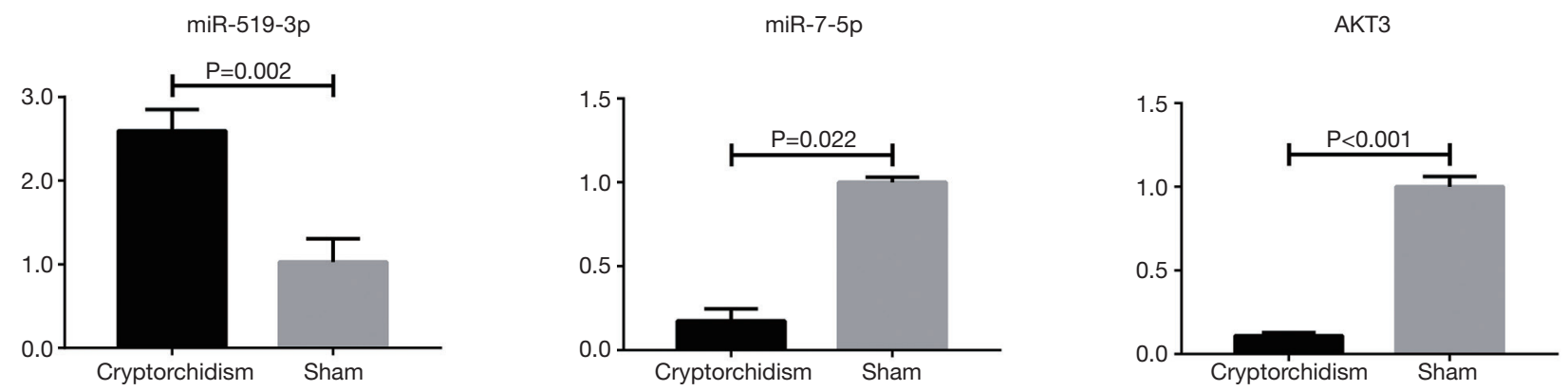

Figure 7 qRT-PCR results showed that the expression levels of miRNA-7-5p (P value $=0.022)$ and AKT3 (P value $<0.001)$ in the testicular tissue of cryptorchidism rats were obviously lower than that of sham surgery controls while miRNA-519d-3p was upregulated $(\mathrm{P}$ value $=0.002)$.

Two miRNAs, hsa-miR-7-5p and hsa-miR-519d-3p, were identified as DE-miRNAs from analysis of GSE149084 and were predicted as miRNAs targeting hub genes. These miRNAs were significantly downregulated in patients with UDTs. In previous studies, overexpression of miRNA-7-5p was found to significantly reduce the migration and invasion of cell lines (41). In contrast, inhibition of miRNA-7-5p expression was found to activate the PI3K/AKT signaling pathway and increase the migration and invasion of cell lines, demonstrating the importance of the miRNA-7-5p/PI3K/ AKT signaling pathway in regulating cell processes (42). As for miR-519d-3p, an increasing number of studies have suggested that miR-519d-3p regulates cell processes in several tumors, including breast cancer (43), gastric cancer (44), and colorectal cancer (45). Studies on the role of miR-7$5 \mathrm{p}$ and miRNA-519d-3p in UDT are scarce. Based on our results, the upregulated miR-519d-3p might bind to the 3'-UTR of AKT3 mRNA and induce its degradation. However, the downregulation of miR-7-5p indicates that a complex mRNA-miRNA interaction chain might be present in patients with UDT. We speculate that the upregulated AKT3 might activate the PI3K-AKT-mTOR signaling pathway to compensate for apoptosis in the early stages of cryptorchidism. As the damage worsens, some miRNAs that target and degrade AKT3 accelerate the pathological damage of cryptorchidism. At this point, the level of AKT3 in the testicular tissue begins to decline. Therefore, we assume that various miRNAs dynamically change during different periods of cryptorchidism and play a regulatory role together.

Infertility related to cryptorchidism is a significant problem in urology. The recommended early correction of UDT does not guarantee future fertility in some cases. Considering this, the assessment of SSC activity based on
AKT3 and its related molecules during surgery can provide a method to predict future fertility.

However, there are two main limitations to our study, and some questions remain. First, our data were not validated in cryptorchidism patients due to a lack of specimens; obtaining samples of cryptorchidism or normal testes is very difficult. Second, when we searched the raw data of GSE25518 in the GEO database, we found that the high infertility risk group and the low one were mixed into one set. But according to the analysis from Hadziselimovic et al., the difference in gene expression between cryptorchid boys in the low infertility risk group and those in the control group is not statistically significant. This is the reason why we could not analyze these groups separately. Because the low infertility risk group and control group both had normal gene expression, we believe that this limitation had little effect on the results.

\section{Conclusions}

In summary, AKT3 likely plays an essential role in regulating SSC self-renewal, proliferation, and differentiation. The PI3K-AKT-mTOR signal pathway functions in SSC maintenance, and alterations in this pathway may explain defects in spermatogenesis. AKT3related miRNAs, including hsa-miR-7-5p and hsa-miR519d-3p, might be responsible for cryptorchidism and cryptorchidism-induced azoospermia and serve as potential biomarkers.

\section{Acknowledgments}

We thank the reviewers and editors for their helpful and 
constructive comments on our work. Also, thanks are due to Limeng Chen for assistance with the experiments and to Chunsheng Hao for valuable discussion.

Funding: None.

\section{Footnote}

Reporting Checklist: The authors have completed the MDAR reporting checklist. Available at https://dx.doi. org/10.21037/tp-21-31

Conflicts of Interest: All authors have completed the ICMJE uniform disclosure form (available at https://dx.doi. org/10.21037/tp-21-31). The authors have no conflicts of interest to declare.

Ethical Statement: The authors are accountable for all aspects of the work in ensuring that questions related to the accuracy or integrity of any part of the work are appropriately investigated and resolved. The study was conducted in accordance with the Declaration of Helsinki (as revised in 2013). Experiments were performed under a project license (No.: SHERLL2019025) granted by the Ethics Committee of the Children's Hospital of Capital Institute of Pediatrics, in compliance with the guidelines for the care and use of animals.

Open Access Statement: This is an Open Access article distributed in accordance with the Creative Commons Attribution-NonCommercial-NoDerivs 4.0 International License (CC BY-NC-ND 4.0), which permits the noncommercial replication and distribution of the article with the strict proviso that no changes or edits are made and the original work is properly cited (including links to both the formal publication through the relevant DOI and the license). See: https://creativecommons.org/licenses/by-nc-nd/4.0/.

\section{References}

1. Radmayr C. Management of undescended testes: European Association of Urology/European Society for Paediatric Urology guidelines. J Pediatr Urol 2017;13:550.

2. Lip SZ, Murchison LE, Cullis PS, et al. A meta-analysis of the risk of boys with isolated cryptorchidism developing testicular cancer in later life. Arch Dis Child 2013;98:20-6.

3. Herrinton LJ, Zhao W, Husson G. Management of cryptorchism and risk of testicular cancer. Am J Epidemiol
2003;157:602-5.

4. Virtanen HE, Toppari J. Cryptorchidism and Fertility. Endocrinol Metab Clin North Am

2015;44:751-60.

5. Docampo MJ, Hadziselimovic F. Molecular Pathology of Cryptorchidism-Induced Infertility. Sex Dev 2015;9:269-78.

6. Hadziselimovic F, Hadziselimovic NO, Demougin P, et al. Testicular gene expression in cryptorchid boys at risk of azoospermia. Sex Dev 2011;5:49-59.

7. Loebenstein M, Thorup J, Cortes D, et al. Cryptorchidism, gonocyte development, and the risks of germ cell malignancy and infertility: A systematic review. J Pediatr Surg 2020;55:1201-10.

8. Kamisawa H, Kojima Y, Mizuno K, et al. Attenuation of spermatogonial stem cell activity in cryptorchid testes. J Urol 2012;187:1047-52.

9. Hadziselimovic F, Herzog B. The importance of both an early orchidopexy and germ cell maturation for fertility. Lancet 2001;358:1156-7.

10. Manku G, Culty M. Mammalian gonocyte and spermatogonia differentiation: recent advances and remaining challenges. Reproduction 2015;149:R139-57.

11. Chen SR, Liu YX. Regulation of spermatogonial stem cell self-renewal and spermatocyte meiosis by Sertoli cell signaling. Reproduction 2015;149:R159-67.

12. Huang $Z$, Tang D, Gao J, et al. miR-34c disrupts spermatogonial stem cell homeostasis in cryptorchid testes by targeting Nanos2. Reprod Biol Endocrinol 2018;16:97.

13. Yao C, Liu Y, Sun M, et al. MicroRNAs and DNA methylation as epigenetic regulators of mitosis, meiosis and spermiogenesis. Reproduction 2015;150:R25-34.

14. Moritoki Y, Hayashi Y, Mizuno K, et al. Expression profiling of microRNA in cryptorchid testes: miR-135a contributes to the maintenance of spermatogonial stem cells by regulating FoxO1. J Urol 2014;191:1174-80.

15. Chen X, Li X, Guo J, et al. The roles of microRNAs in regulation of mammalian spermatogenesis. J Anim Sci Biotechnol 2017;8:35.

16. Holt JE, Stanger SJ, Nixon B, et al. Non-coding RNA in Spermatogenesis and Epididymal Maturation. Adv Exp Med Biol 2016;886:95-120.

17. Hamer G, de Rooij DG. Mutations causing specific arrests in the development of mouse primordial germ cells and gonocytes. Biol Reprod 2018;99:75-86.

18. Hadziselimovic F, Hadziselimovic NO, Demougin P, 
et al. EGR4 is a master gene responsible for fertility in cryptorchidism. Sex Dev 2009;3:253-63.

19. Li Z, Liu J, Wang W, et al. Investigation of hub genes involved in diabetic nephropathy using biological informatics methods. Ann Transl Med 2020;8:1087.

20. Huang da W, Sherman BT, Lempicki RA. Systematic and integrative analysis of large gene lists using DAVID bioinformatics resources. Nat Protoc 2009;4:44-57.

21. Shannon P, Markiel A, Ozier O, et al. Cytoscape: a software environment for integrated models of biomolecular interaction networks. Genome Res 2003;13:2498-504.

22. Szklarczyk D, Gable AL, Lyon D, et al. STRING v11: protein-protein association networks with increased coverage, supporting functional discovery in genome-wide experimental datasets. Nucleic Acids Res 2019;47:D607-13.

23. Chin $\mathrm{CH}$, Chen SH, Wu HH, et al. cytoHubba: identifying hub objects and sub-networks from complex interactome. BMC Syst Biol 2014;8 Suppl 4:S11.

24. Bai Q, Liu H, Guo H, et al. Identification of Hub Genes Associated With Development and Microenvironment of Hepatocellular Carcinoma by Weighted Gene Coexpression Network Analysis and Differential Gene Expression Analysis. Front Genet 2020;11:615308.

25. Dweep H, Sticht C, Pandey P, et al. miRWalk--database: prediction of possible miRNA binding sites by "walking" the genes of three genomes. J Biomed Inform 2011;44:839-47.

26. Nassau DE, Chu KY, Blachman-Braun R, et al. The pediatric patient and future fertility: optimizing longterm male reproductive health outcomes. Fertil Steril 2020;113:489-99.

27. Papaioannou MD, Nef S. microRNAs in the testis: building up male fertility. J Androl 2010;31:26-33.

28. Barthold JS. Undescended testis: current theories of etiology. Curr Opin Urol 2008;18:395-400.

29. Barthold JS, Reinhardt S, Thorup J. Genetic, Maternal, and Environmental Risk Factors for Cryptorchidism: An Update. Eur J Pediatr Surg 2016;26:399-408.

30. Lee J, Kanatsu-Shinohara M, Inoue K, et al. Akt mediates self-renewal division of mouse spermatogonial stem cells. Development 2007;134:1853-9.

31. Ishii K, Kanatsu-Shinohara M, Toyokuni S, et al. FGF2 mediates mouse spermatogonial stem cell self-renewal via upregulation of Etv 5 and Bcl6b through MAP2K1 activation. Development 2012;139:1734-43.

32. Zhang Y, Wang S, Wang X, et al. Endogenously produced
FGF2 is essential for the survival and proliferation of cultured mouse spermatogonial stem cells. Cell Res 2012;22:773-6.

33. Hoxhaj G, Manning BD. The PI3K-AKT network at the interface of oncogenic signalling and cancer metabolism. Nat Rev Cancer 2020;20:74-88.

34. Mirdamadi Y, Bommhardt U, Goihl A, et al. Insulin and Insulin-like growth factor- 1 can activate the phosphoinositide-3-kinase /Akt/FoxO1 pathway in T cells in vitro. Dermatoendocrinol 2017;9:e1356518.

35. Liu Z, Zhou K, Fu W, et al. Insulin-Like Growth Factor 1 Activates PI3k/Akt Signaling to Antagonize Lumbar Disc Degeneration. Cell Physiol Biochem 2015;37:225-32.

36. Li R, Azzollini D, Shen R, et al. Postnatal germ cell development during first 18 months of life in testes from boys with non-syndromic cryptorchidism and complete or partial androgen insensitivity syndrome. J Pediatr Surg 2019;54:1654-9.

37. Grabinski N, Bartkowiak K, Grupp K, et al. Distinct functional roles of Akt isoforms for proliferation, survival, migration and EGF-mediated signalling in lung cancer derived disseminated tumor cells. Cell Signal 2011;23:1952-60.

38. Paul-Samojedny M, Pudełko A, Kowalczyk M, et al. Combination Therapy with AKT3 and PI3KCA siRNA Enhances the Antitumor Effect of Temozolomide and Carmustine in T98G Glioblastoma Multiforme Cells. BioDrugs 2016;30:129-44.

39. Yu JS, Cui W. Proliferation, survival and metabolism: the role of PI3K/AKT/mTOR signalling in pluripotency and cell fate determination. Development 2016;143:3050-60.

40. Cobellis G, Noviello C, Nino F, et al. Spermatogenesis and cryptorchidism. Front Endocrinol (Lausanne) 2014;5:63.

41. Giles KM, Brown RA, Epis MR, et al. miRNA-7-5p inhibits melanoma cell migration and invasion. Biochem Biophys Res Commun 2013;430:706-10.

42. Shukla A, Gupta P, Singh R, et al. Glycolytic inhibitor 2-Deoxy-d-Glucose activates migration and invasion in glioblastoma cells through modulation of the miR-7-5p/ TFF3 signaling pathway. Biochem Biophys Res Commun 2018;499:829-35.

43. Li D, Song H, Wu T, et al. MiR-519d-3p suppresses breast cancer cell growth and motility via targeting LIM domain kinase 1. Mol Cell Biochem 2018;444:169-78.

44. Li YY, Shao JP, Zhang SP, et al. miR-519d-3p Inhibits 
Cell Proliferation and Invasion of Gastric Cancer by Downregulating B-Cell Lymphoma 6. Cytogenet Genome Res 2018;154:12-9.

Cite this article as: Jia H, Ma T, Jia S, Ouyang Y. AKT3 and related molecules as potential biomarkers responsible for cryptorchidism and cryptorchidism-induced azoospermia. Transl Pediatr 2021;10(7):1805-1817. doi: 10.21037/tp-21-31
45. Ye X, Lv H. MicroRNA-519d-3p inhibits cell proliferation and migration by targeting TROAP in colorectal cancer. Biomed Pharmacother 2018;105:879-86. 